\title{
O Prático Visual: o Infográfico como Ferramenta Facilitadora do Aprendizado no Curso de Medicina
}

\author{
The Visual Practice: the Infographic as a Facilitating Tool for \\ Learning in Medical School
}

\author{
Diêgo Andrade de Oliveira ${ }^{I}$ (i) \\ Rosângela Souza Lessa ${ }^{I}(\mathbb{D}$ \\ Suzana Cristina Silva Ribeiro ${ }^{I}$ (iD \\ Pedro Fonseca de Vasconcelos ${ }^{I}$ (iD
}

\section{PALAVRAS-CHAVE}

- Educação Médica.

- Aprendizagem Baseada em Problemas.

- Conhecimento.

- Criatividade

Introdução: No contexto das graduações médicas, o desenvolvimento de metodologias que estimulam a busca do aprendizado, a autonomia e a criatividade dos discentes é essencial para a formação médica no Brasil. O estudo visa descrever a construção de infográficos como proposta pedagógica para o aprendizado dos processos orgânicos do envelhecimento humano por estudantes de Medicina. Método: Os discentes do quarto período do curso de Medicina de uma instituição de ensino superior construíram infográficos como requisito de conteúdo prático do módulo "Processos de Envelhecimento". Adotou-se o infográfico do tipo estático, seguindo critérios como: definição do público-alvo; definição do objetivo; escolha do tema; seleção das informações mais relevantes (foco); linguagem direta e acessível; informações organizadas; escolhas das paletas de cores e estilo; e esboço do infográfico. Todo processo de elaboração foi supervisionado pelo docente responsável, sendo previamente estabelecidos os critérios de avaliação. Resultados: A turma foi dividida em sete grupos, resultando na produção de um infográfico com uma temática específica por grupo. Os temas do envelhecimento humano foram: doenças articulares degenerativas, enfraquecimento ósseo, pneumonia no idoso, infarto agudo do miocárdio, demência vascular, aterosclerose e herpes-zóster. É válido salientar que, além da criação, cada grupo apresentou aos demais colegas o produto final, explicitando cada item inserido no infográfico estático. Conclusões: Foi possível observar que os alunos cumpriram de forma satisfatória os requisitos avaliativos propostos, demonstrando envolvimento na construção dos infográficos e, sobretudo, no aprendizado simples, criativo e objetivo, utilizando uma poderosa ferramenta visual. Acrescenta-se também que o material impresso servirá de apoio no laboratório de histologia e em atividades extramuros. 


\section{KEYWORDS}

- Education Medical.

- Problem-Based Learning.

- Knowledge.

- Creativity.

Recebido em 19/5/20

Aceito em $1 / 7 / 20$

\section{INTRODUÇÃO}

$\mathrm{Na}$ área da educação, muito se discute sobre melhorias nas práticas de ensino existentes, o que inclui repensar a formação de professores e a reformulação de currículos, a fim de promover um ensino em que o discente possa ser ativo e participativo em seu papel, desenvolvendo autonomia e criticidade no processo de aprendizagem ${ }^{1,2}$, além de ter a possibilidade de explorar a produção do conhecimento e a transformação dos paradigmas educacionais por meio do fortalecimento da criatividade ${ }^{3}$. Nesse cenário, o professor deixa de ser o centro do conhecimento e tornase um mediador, um instigador no processo de aprendizagem, responsável por se valer de diferentes recursos para proporcionar aos discentes uma estratégia pedagógica construtiva ${ }^{1,2}$.

No contexto do ensino médico, a proposta de basear o aprendizado do aluno em metodologias ativas começa a ser normatizada no Brasil, em 2001, nas Diretrizes Curriculares Nacionais (DCN) voltadas para os cursos de Medicina e tornou-se obrigatória nas DCN de 2014, em que se priorizam as propostas pedagógicas que visem ao "aprender a aprender". Desse modo, as DCN proporcionam uma atuação ímpar e ativa de cada discente, valem-se das atividades individuais e coletivas e desenvolvem não só o conhecimento teórico, mas também as relações interpessoais, a reflexão dos conteúdos, isto é, uma preparação constante para a busca do conhecimento ${ }^{4-7}$.

Não obstante isso, é salutar o uso de ferramentas que atuem como modificadoras do modelo tradicional de educação vigente na sociedade, de forma a garantir a efetivação dos princípios metodológicos de uma prática educativa transformadora. Assim, a infografia surge como um instrumento para auxiliar nessa mudança conferindo engajamento de todos os envolvidos no processo de sua construção. O infográfico é uma ferramenta utilizada para sintetizar as informações apresentadas em um texto. É uma explicação feita pela associação de textos e imagens (fotografia, desenho, gráficos, anagramas, esquemas, entre outros) cuja proposta é mostrar a informação concisa de forma atraente, clara e objetiva para o

\section{ABSTRACT}

Introduction: In the context of medical school, the development of methodologies that stimulate the students' describe the construction of infographics as a pedagogical proposal for the learning of organic human aging processes by medical students. Method: Medical students attending the $4^{\text {th }}$ period at a Higher Education Institution built infographics, as a requirement for the practical content of the Aging Process module. The static-type infographic was adopted, following criteria such as the definition of the target audience; definition of the objective; choice of topic; selection of the most relevant information (focus); direct and accessible language organized information; choices of color palettes and style and; infographic sketch. The entire creation process was supervised by the teacher in charge of the project, and evaluation criteria were previously established. Results The class was divided into seven groups, resulting in the production of an infographic with a specific topic per group. The human aging topics were: Degenerative Joint Diseases, Bone Weakness, Pneumonia in the Elderly, addition to the creation, each group presented the final product to the other colleagues, explaining each item included in the static infographic. Conclusions: We observed that the students satisfactorily met the proposed evaluation requirements, demonstrating their involvement in the construction of infographics and, above all, in simple, creative and objective learning, using a powerful visual tool. We also add that the printed material will be used as aid in the histology laboratory and in extramural activities. 
passo a passo que indica os elementos fundamentais para a criação dessa ferramenta: definição do público-alvo; definição do objetivo; escolha do tema; seleção das informações mais relevantes (foco); linguagem direta e acessível; informações organizadas; escolhas das paletas de cores e estilo; e esboço do infográfico ${ }^{12}$.

Adotou-se o infográfico do tipo estático ${ }^{3}$, que se caracteriza pela fusão de textos e imagens (fotografia, ilustração), como padrão para o desenvolvimento das temáticas. Para abordar os conteúdos, cada grupo pôde escolher um tópico específico, de acordo com as temáticas sorteadas, dispostas no Quadro 1, explanando o processo de envelhecimento do órgão ou sistema em questão, com foco nas alterações histológicas, bem como suas alterações fisiopatológicas.

Mediante a proposta das temáticas apresentadas para a criação dos infográficos, solicitou-se que os discentes norteassem sua produção a partir dos seguintes elementos: título e subtítulo; definição; fatores de risco; quadro clínico; diagnóstico; medidas de prevenção e tratamentos. Posteriormente, cada grupo ficou responsável por apresentar o seu infográfico para os demais discentes da turma, detalhando o processo de envelhecimento relacionado ao seu tema, bem como as alterações histológicas provenientes do processo de envelhecimento fisiológico ou patológico, em conformidade com os critérios anteriormente elencados. Tal proposta teve o objetivo de socialização e compartilhamento de

\section{Quadro 1}

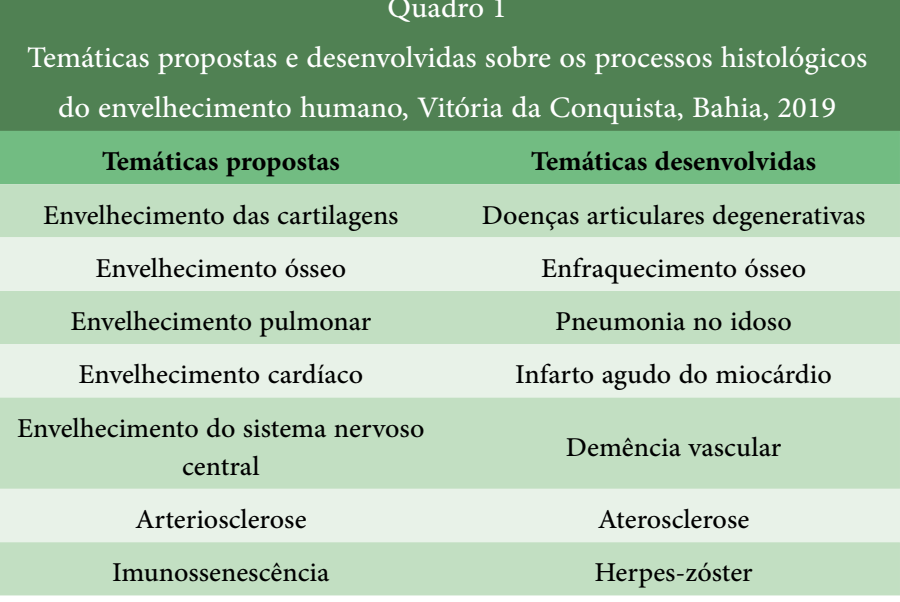

Fonte: Elaborada pelos autores.

\section{Quadro 3}

Critérios de avaliação dos discentes na construção dos infográficos, Vitória da Conquista, Bahia, 2019

Critérios estabelecidos para avaliação dos infográficos

Participação nos encontros preestabelecidos com o professor.

Atuação como membro ativo (desenvolvimento de atividades propostas).

Apresentação das tarefas propostas nos prazos estabelecidos.

Autoria (imagens e textos).

Infográfico (informações claras, objetivas, coerentes, confiáveis; grafia correta das palavras; linguagem científica; layout adequado).

Referências bibliográficas (atuais e confiáveis).

Domínio e clareza na apresentação oral do infográfico. todo material desenvolvido ao longo do semestre, favorecendo, assim, a reflexão individual e coletiva, bem como o aprendizado de todos os temas propostos, e não somente o que cada grupo desenvolveu. Acrescenta-se também que o produto final de cada infográfico produzido foi impresso em formato de banner e disposto no Laboratório de Histologia da IES, permitindo o acesso fácil e servindo como material de apoio para todos os discentes da IES.

É válido salientar que todo processo para criação dos infográficos teve o acompanhamento do professor supervisor desde a concepção, a fase de pesquisa, o esboço do layout até a finalização, e cada grupo deveria comparecer aos nove encontros pré-agendados, conforme cronograma detalhado no Quadro 2.

Além das etapas de elaboração, o docente estabeleceu critérios para o processo avaliativo, já que a criação dos infográficos estava intimamente ligada ao componente prática do módulo de envelhecimento. Dessa forma, a avaliação se deu de forma processual e contínua, de modo que cada discente fosse avaliado individual e coletivamente de acordo com os critérios estabelecidos no Quadro 3, permitindo ainda o feedback ao final do processo.

Os discentes desenvolveram sete infográficos (Figura 1) que permearam diferentes sistemas do organismo, conforme consta no Quadro 1, e permitiram uma visão global do processo de envelhecimento, desde o entendimento da fisiopatologia, os fatores de risco, a apresentação

\begin{tabular}{|c|c|c|}
\hline \multicolumn{3}{|c|}{$\begin{array}{l}\text { Quadro } 2 \\
\text { Cronograma de acompanhamento das atividades na elaboração dos } \\
\text { infográficos, Vitória da Conquista, Bahia, } 2019\end{array}$} \\
\hline Atividades & Grupos & Encontros/horários \\
\hline $\begin{array}{l}\text { Explanação sucinta dos temas a } \\
\text { serem abordados no módulo. }\end{array}$ & $\begin{array}{l}1,2,3 \text { e } 4 \\
5,6 \text { e } 7\end{array}$ & $\begin{array}{l}1^{\circ} \\
\text { Das } 8 \text { às } 12 \mathrm{~h} \\
2^{\circ} \\
\text { Das } 8 \text { às } 12 \mathrm{~h}\end{array}$ \\
\hline $\begin{array}{l}\text { Apresentação dos temas a serem } \\
\text { abordados, divisão dos grupos e } \\
\text { organização das tarefas por eles. }\end{array}$ & $\begin{array}{l}1,2,3 \text { e } 4 \\
5,6 \text { e } 7\end{array}$ & $\begin{array}{l}3^{\circ} \\
\text { Das } 8 \text { às } 12 \mathrm{~h} \\
4^{\circ} \\
\text { Das } 8 \text { às } 12 \mathrm{~h}\end{array}$ \\
\hline $\begin{array}{l}\text { Encontro com os grupos: } \\
\text { apresentação da estrutura e do } \\
\text { layout dos infográficos e da } \\
\text { bibliografia utilizada. }\end{array}$ & $\begin{array}{l}1,2,3 \text { e } 4 \\
5,6 \text { e } 7\end{array}$ & $\begin{array}{l}5^{\circ} \\
\text { Das } 8 \text { às } 12 \mathrm{~h} \\
6^{\circ} \\
\text { Das } 8 \text { às } 12 \mathrm{~h}\end{array}$ \\
\hline $\begin{array}{l}\text { Encontro com os grupos: estrutura } \\
\text { final dos infográficos. }\end{array}$ & $1,2,3$ e 4 & $\begin{array}{l}7^{\mathrm{o}} \\
\text { Das } 8 \text { às } 10 \mathrm{~h} \\
8^{\circ} \\
\text { Das } 10 \text { às } 12 \mathrm{~h}\end{array}$ \\
\hline $\begin{array}{c}\text { Apresentação dos infográficos por } \\
\text { grupo. }\end{array}$ & Todos & $\begin{array}{c}9^{\circ} \\
\text { Das } 19 \text { às } 22 \mathrm{~h}\end{array}$ \\
\hline
\end{tabular}

Fonte: Elaborada pelos autores.

Fonte: Elaborada pelos autores.

$3 \mid$\begin{tabular}{l|l} 
REVISTA BRASILEIRA DE EDUCAÇÃO MÉDICA \\
\hline 44 (4) : e109; 2020
\end{tabular} 


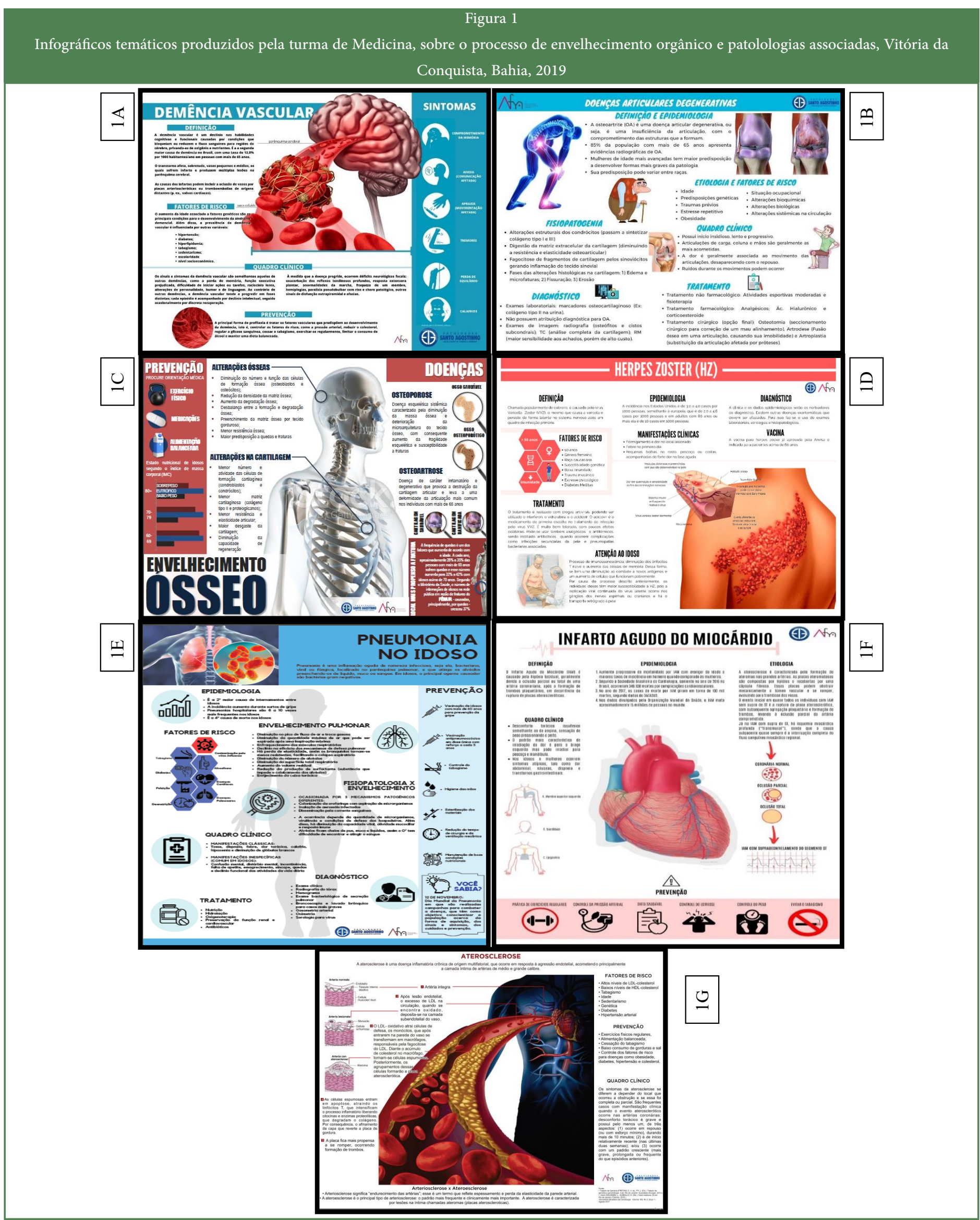

1A: Infográfico sobre o envelhecimento do sistema nervoso central (demência vascular); 1B: infográfico sobre o envelhecimento das cartilagens (doenças articulares degenerativas); 1C: infográfico sobre o envelhecimento ósseo (enfraquecimento ósseo); 1D: infográfico sobre a imunossenescência (herpes-zóster); 1E: infográfico sobre o envelhecimento pulmonar (pneumonia no idoso); 1F: infográfico sobre o envelhecimento cardíaco (infarto agudo do miocárdio); e 1G: infográfico sobre arteriosclerose (aterosclerose). 
dos sinais e sintomas clínicos e as medidas de tratamento até as abordagens preventivas que são trabalhadas por meio da educação em saúde.

A escolha do infográfico como ferramenta facilitadora do processo ensino-aprendizagem foi feita com o objetivo de explorar suas potencialidades, entre as quais: 1 . o seguimento de cada etapa do processo de criação, conferindo acompanhamento no processo de aprendizagem do aluno, uma vez que explorou, quando necessário, cada passo na construção do infográfico; 2 . desenvolvimento de habilidades cognitivas, tais como a interpretação, análise e síntese; 3 . incentivo à participação nos encontros para a construção do infográfico, por conta de seu caráter dinâmico, atrativo e objetivo, estimulando o senso de responsabilidade, liderança e trabalho em equipe; 4. desenvolvimento de uma nova perspectiva de observação, de modo que os discentes se percebessem aspectos fundamentais da temática abordada, levando em consideração o problema proposto em sua magnitude; 5. possibilidade de associação de diferentes recursos tecnológicos na construção do infográfico, além do desenvolvimento de estratégias de exposição de informações, fundamentais para a rotina de um profissional da área de saúde?.

Como já mencionado, a infografia, no processo de construção, configura-se como uma ferramenta que possibilita ao aluno desenvolver diferentes formas de expressar sua compreensão da temática trabalhada. O caráter multifacetado pode apresentar diversas possibilidades ao ser estruturado, pois cada indivíduo apresenta um olhar específico e relaciona-o conforme sua interpretação ${ }^{3}$. Sendo assim, pode-se observar que os alunos cumpriram os requisitos estabelecidos para os critérios avaliativos propostos, demonstrando envolvimento na construção do material, e, embora tenhamos observado dificuldade em montar os grupos de trabalho, foi possível superar tal limitação e desenvolver habilidades de trabalho em equipe.

A literatura aponta o infográfico como uma potente ferramenta da metodologia ativa para que o discente possa, por meio da comunicação gráfica visual, construir o conhecimento de forma criativa e informativa ${ }^{3,10}$, além de permitir uma avaliação do aluno de forma processual e contínua. Por fim, vale ressaltar que o presente relato apresenta essa ferramenta como um dispositivo avaliativo que compreende o sujeito avaliado em todas as suas dimensões.

\section{CONSIDERAÇÕES FINAIS}

A infografia foi uma ferramenta utilizada com êxito para a construção do conhecimento dos discentes de Medicina, pois permitiu o aprendizado individual e coletivo, a criatividade, a reflexão, a objetividade e a utilização da linguagem gráfica para o entendimento dos processos orgânicos do envelhecimento.

Além do aprendizado dos discentes que elaboraram os infográficos, o produto final servirá como instrumento de aquisição de conhecimento objetivo para os alunos que frequentarão o laboratório de histologia, podendo também ser utilizado fora do contexto acadêmico, de modo a possibilitar a divulgação de informações por meio dessa ferramenta e da produção acadêmica.

Acrescenta-se ainda a importância da infografia como dispositivo prático de avaliação, funcionando como uma ferramenta alternativa para o processo avaliativo, visto que, nesse modelo, a avaliação se dá de forma processual e contínua, levando em consideração as diferentes facetas e habilidades envolvidas e desenvolvidas por cada discente.

\section{REFERÊNCIAS}

1. Cezar PHN, Guimarães FT, Gomes AP, Rôças G, Siqueira-Batista R. Transição paradigmática na educação médica: um olhar construtivista dirigido à aprendizagem baseada em problemas. Rev Bras Educ Med. 2010;34(2):298-303.

2. Bedin FC, Kiouranis NMM, Sari ERG, Brustolin LC. Construção de infográficos como uma ferramenta potencializadora do educar pela pesquisa. Arquivos do Mudi. 2017;21(3):26-37.

3. Bottentuit Júnior JB, Mendes AGLM, Da Silva NM. O uso do infográfico em sala de aula: uma experiência na disciplina de literatura. Revista Educa. 2017;11(3)105-27.

4. Brasil. Ministério da Educação. Conselho Nacional de Educação. Câmara de Educação Superior. Resolução nº CNE/CES 4/2001. Institui Diretrizes Curriculares Nacionais do Curso de Graduação em Medicina. Diário Oficial da União Brasília, 9 nov 2001; Seção 1, p. 38.

5. Brasil. Ministério da Educação. Conselho Nacional de Educação. Câmara da Educação Superior. Resolução nº CNE/CES 3/2014. Institui Diretrizes Curriculares Nacionais do Curso de Graduação em Medicina e dá outras providências. Diário Oficial da União Brasília, 23 jun 2014; Seção 1, p. 8-11.

6. Franco CAGS, Cubas MR, Franco RS. Currículo de medicina e as competências propostas pelas diretrizes curriculares. Rev Bras Educ Med. 2014;38(2):221-30.

7. Rocha VXM. Reformas na educação médica no Brasil: estudo comparativo entre as diretrizes curriculares nacionais dos cursos de graduação em medicina de 2001 e 2014 [dissertação]. Santos: Universidade Católica de Santos; 2017.

8. Pedroza NP, Bezerra EP, Nicolau MA. Ferramentas para elaboração de infográficos: um estudo de caso. Anais do X Encontro Virtual de Documentação em Software Livre e VII Congresso Internacional de Linguagem e Tecnologia Online; 2013 nov. 11-13; Belo Horizonte, Brasil. Belo Horizonte: UFMG; 2013. p. 1-8.

9. Lyra KT, Oliveira RNB, Reis RCD, Cruz WM, Nakagawa EY, Isotani S. Infográficos versus materiais de aprendizagem tradicionais: uma investigação empírica. Renote. 2016;14(2):1-12.

10. Bottentuitt Júnior JB, Lisboa ES, Coutinho CP. O infográfico e as suas potencialidades educacionais. Quaestio - Revista de Estudos em Educação. 2011;13(2):163-83.

11. Pinto CL, Tavares HM. O lúdico na aprendizagem: apreender e aprender. Revista da Católica. 2010;2(3):226-35.

12. Nogueira A. Crie o seu infográfico com essas ferramentas gratuitas! Hotmart; 2019 [acesso em 7 fev 2020]. Disponível em: https://blog. hotmart.com/pt-br/como-fazer-um-infografico/.

\section{CONTRIBUIÇÃO DOS AUTORES}

Diêgo Andrade de Oliveira, idealizador do projeto e professor responsável pela intervenção, participou de todo o planejamento, da construção científica, execução, análise, escrita e redação final do manuscrito. Rosângela Souza Lessa contribuiu na proposta da ideia de intervenção, elaboração, escrita, discussão do relato e revisão da redação final do manuscrito. Suzana Cristina Silva Ribeiro contribuiu na análise, escrita e revisão da redação final do manuscrito. Pedro Fonseca de 
Vasconcelos contribuiu na proposta da ideia de intervenção, elaboração, escrita, discussão do relato e revisão da redação final do manuscrito.

\section{CONFLITO DE INTERESSES}

Os autores declaram não haver conflito de interesses neste estudo.

\section{ENDEREÇO PARA CORRESPONDÊNCIA}

Diêgo Andrade de Oliveira. Faculdades Santo Agostinho, Avenida Olívia Flores, 200, Candeias, Vitória da Conquista, BA, Brasil. CEP: 45028-100.

E-mail: diego.andrade@fasa.edu.br 